\title{
Direct Downconversion of Multiband RF Signals Using Bandpass Sampling
}

\author{
Ching-Hsiang Tseng and Sun-Chung Chou
}

\begin{abstract}
Bandpass sampling can be used by radio receivers to directly digitize the radio frequency (RF) signals. Although the bandpass sampling theory for single-band RF signals is well established, its counterpart for multiband RF signals is relatively immature. In this paper, we propose a novel and efficient method to find the ranges of valid bandpass sampling frequency for direct downconverting multiband RF signals. Simple formulas for the ranges of valid bandpass sampling frequency in terms of the frequency locations of the multiple RF bands are derived. The result can be used to design a multiband receiver for software defined radios.
\end{abstract}

Index Terms - Software defined radio, bandpass sampling, sampling methods, analog-digital conversion, signal sampling.

\section{INTRODUCTION}

$\mathbf{M}$ OBILE communication standards emerged in an increasing speed in recent years. The software defined radio (SDR) [1], which was originally conceived for military applications [2], has the agility of changing its functionality through replacement of the application program and hence provides a relatively economical way to accommodate the rapid emerging mobile communication standards. The main philosophy in designing a SDR receiver front end is to push the analog-to-digital converter (ADC) as close to the antenna as possible. The ultimate goal, would be direct digitizing the radio frequency (RF) signal at the receiver antenna output [3]. To achieve this goal, one can manage to use a wideband highspeed ADC to convert the RF signal to digital. However, the required sampling rate for the $\mathrm{ADC}$ could often be too high to be attained if the Nyquist sampling theorem is to be satisfied [4]. Alternatively, being aware of the bandpass nature of the radio signal, one can use the bandpass sampling [5] instead to directly downconvert the desired RF signal from the RF to an intermediate frequency (IF). This can significantly relax the demand for fast ADCs and digital signal processors (DSPs) in a SDR design.

In military and commercial SDRs, there is a need for a single radio to communicate simultaneously with many radios using different RF bands and different modulation schemes [2], [6], [7], [8]. To attain this goal, methods for direct downconversion of multiband RF signals using a single ADC

Manuscript received August 11, 2003; revised November 13, 2003; accepted January 11, 2005. The associate editor coordinating the review of this letter and approving it for publication was T. Boucouvalas. This paper was presented in part at the IEEE International Conference on Communications (ICC'03), Anchorage, AK, May 2003.

C.-H. Tseng is with the Department of Electrical Engineering, Nationa Taiwan Ocean University, 2 Pei-Ning Rd., Keelung, 202, Taiwan (e-mail: chtseng@mail.ntou.edu.tw).

S.-C. Chou is with Wistron Corp., 21F, 88, Sec. 1, Hsin Tai Wu Rd., Hsichih, Teipei Hsien, 221, Taiwan (e-mail: eddie_chou@wistron.com.tw).

Digital Object Identifier 10.1109/TWC.2006.01012.

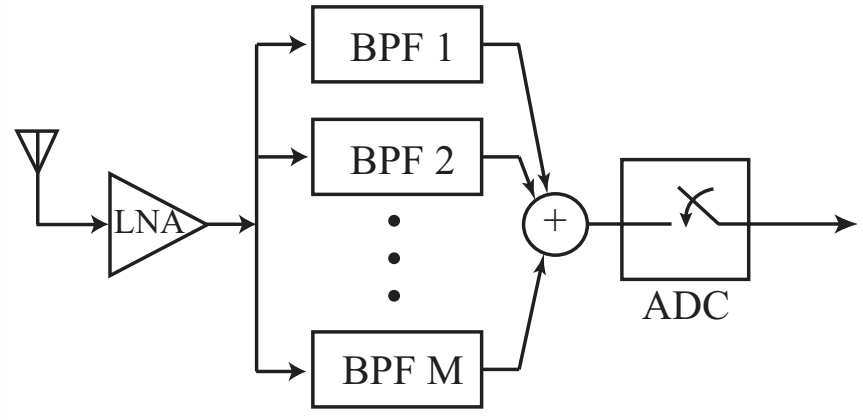

(a)

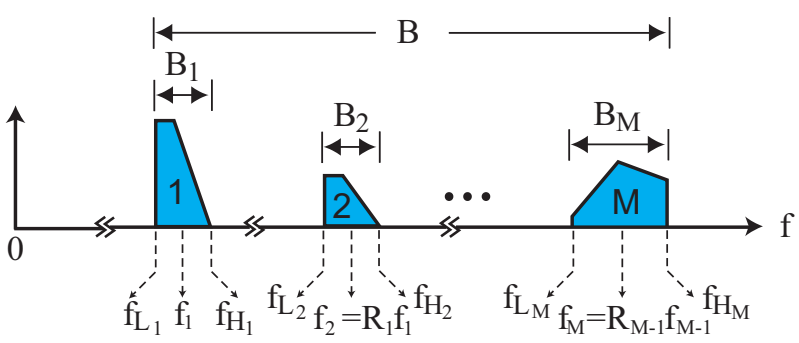

(b)

Fig. 1. (a) The SDR receiver front end. (b) The spectrum of the multiband $\mathrm{RF}$ signal at the input of the ADC.

may be appreciated. One possible SDR receiver front end design fulfilling this purpose is shown in Fig. 1(a) [9], where the input signal to the ADC would be a multiband RF signal whose spectrum is like that shown in Fig. 1(b). A method to determine the bandpass sampling frequency for the ADC in Fig. 1(a) has been proposed in [9]. This method, however, is computationally intensive. Its computational complexity can be alleviated to certain extent by using the method presented in [10].

In this paper, a novel method is proposed to obtain the valid bandpass sampling frequency ranges for direct downconversion of multiband RF signals. In the proposed method, we first observe that a valid sampling frequency must not cause aliasing in the sampled signal. To be immune from aliasing, the spectral replicas (which are produced by sampling) of the signals at different bands must be positioned in frequency in a particular order without overlapping in the sampled signal spectrum. By analyzing the constraints on the sampling frequency for all the possible orders of spectral replicas, we derive an efficient procedure for finding the valid sampling frequency ranges. Compared to the conventional approach, the proposed method is superior in the sense of both computational complexity and ease of implementation. Computer simulation on direct downconversion of multiband 


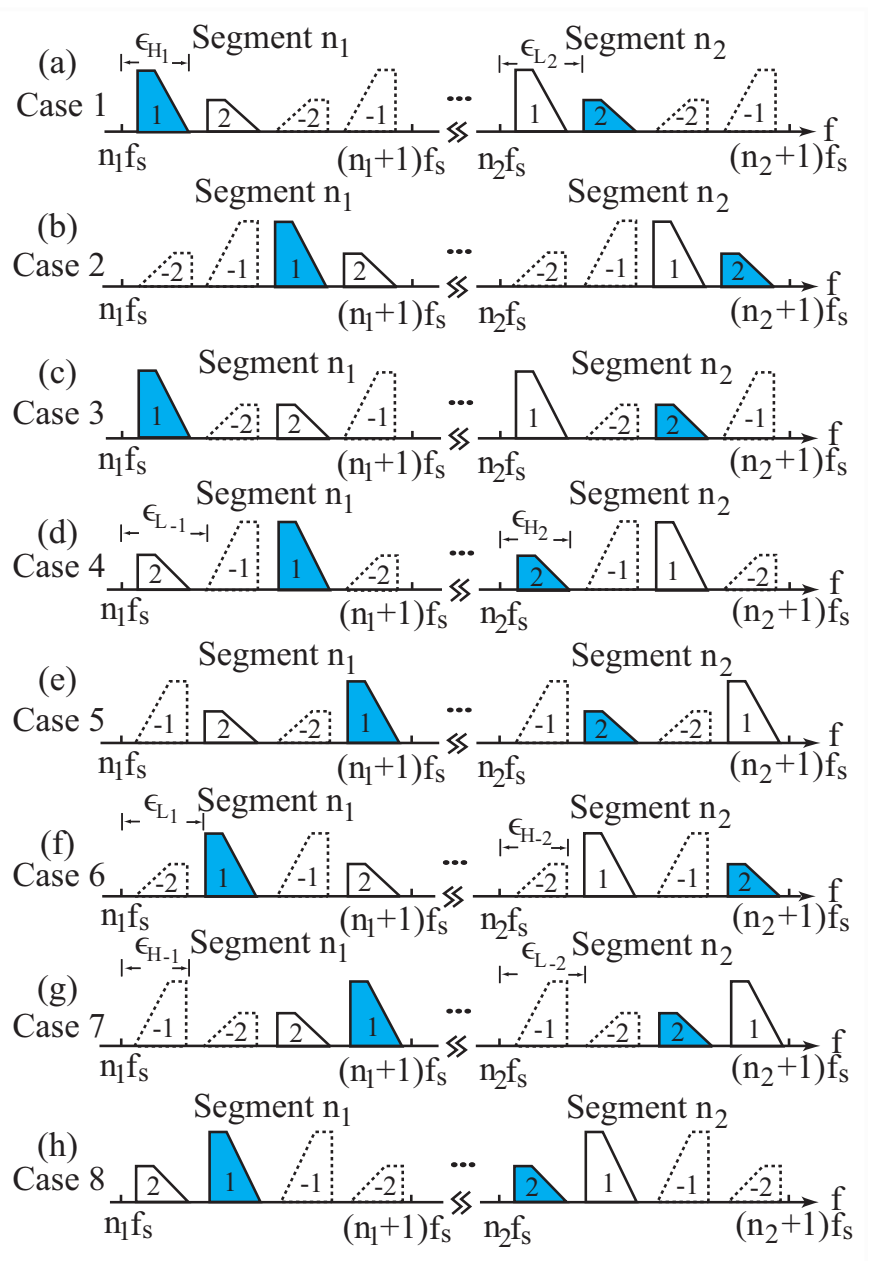

Fig. 2. The dual-band signal shown in Fig. 1(b) (assuming $M=2$ ) after bandpass sampling. The 8 possible replica orders are shown in (a) to (h). The parameters $\epsilon_{L_{i}}$ and $\epsilon_{H_{i}}(i= \pm 1, \pm 2)$ are defined in (a), (d), (f), and (g).

GSM signals is conducted to demonstrate the usage of the proposed method.

\section{BANDPASS SAMPLING DUAL-BAND RF SignAlS}

Consider the problem of sampling a dual-band RF signal whose spectrum is like that shown in Fig. 1(b) with $M=2$. To be immune from aliasing, the sampling frequency $f_{s}$ needs to be chosen without causing spectral overlapping in the sampled signal spectrum. This leads to the 8 possible replica orders shown in Fig. 2, where we use shaded trapezoids to denote the spectrum of the original signal, and solid and dashed trapezoids to denote the replicas for positive-frequency and negative-frequency spectra, respectively.

For a given replica order, the sampling frequency must satisfy two types of constraints: one is referred to as the neighbor constraint and the other is referred to as the boundary constraint in this paper. Note that, as shown in Figs. 2(a) and 2(b), the replicas ' 1 ' and ' 2 ' are neighbors in the first half of a segment in Case 1 and in the second half of a segment in Case 2 . Since the positions of replica ' $i$ ' and ' $-i$ ' are symmetric with respective to the midpoint of a segment, one can also see from Figs. 2(a) and 2(b) that the replicas '-2' and '-1' are neighbors in the second half of a segment in Case 1 and in the
TABLE I

THE NEIGHBor CONSTRAINTS FOR THE 8 CASES IN FIG. 2.

\begin{tabular}{|c|c|c|c|c|}
\hline Group & Neighbors & Case & Shorthand & Constraint \\
\hline 1 & $\begin{array}{l}\langle 1,2\rangle \\
\langle-2,-1\rangle\end{array}$ & $\begin{array}{l}1 \\
2\end{array}$ & $\epsilon_{H_{1}} \leq \epsilon_{L_{2}}$ & $f_{s} \leq \frac{f_{L_{2}}-f_{H_{1}}}{n_{2}-n_{1}}$ \\
\hline 2 & $\begin{array}{l}\langle 1,-2\rangle \\
\langle 2,-1\rangle\end{array}$ & $\begin{array}{l}3 \\
4 \\
\end{array}$ & $\epsilon_{H_{1}} \leq \epsilon_{L_{-2}}$ & $f_{s} \geq \frac{f_{H_{1}}+f_{H_{2}}}{n_{1}+n_{2}+1}$ \\
\hline 3 & $\begin{array}{l}\langle-1,2\rangle \\
\langle-2,1\rangle\end{array}$ & $\begin{array}{l}5 \\
6\end{array}$ & $\epsilon_{H_{-1}} \leq \epsilon_{L_{2}}$ & $f_{s} \leq \frac{f_{L_{1}}+f_{L_{2}}}{n_{1}+n_{2}+1}$ \\
\hline 4 & $\begin{array}{l}\langle-1,-2\rangle \\
\langle 2,1\rangle\end{array}$ & $\begin{array}{l}7 \\
8\end{array}$ & $\epsilon_{H_{-1}} \leq \epsilon_{L_{-2}}$ & $f_{s} \geq \frac{f_{H_{2}}-f_{L_{1}}}{n_{2}-n_{1}}$ \\
\hline
\end{tabular}

first half of a segment in Case 2. If those cases having the same neighboring replicas in either the first or the second half of a segment are considered as in one group, we may categorize the 8 cases in Fig. 2 into 4 groups, as shown in Table I, where the neighboring relation between two replicas $j$ and $k$ is denoted by $\langle j, k\rangle$. The two cases in each group have the same neighboring replicas and thus share the same neighbor constraint. However, they have different boundary constraints because the neighboring replicas show up in different halves of a segment.

For simplicity, we define $\epsilon_{L_{i}}$ and $\epsilon_{H_{i}}(i= \pm 1, \pm 2)$ as the relative lowest and highest frequencies of replica ' $i$ ' in a segment, as shown in Figs. 2(a), 2(d), 2(f), and 2(g). By comparing Figs. 2(a), 2(d), 2(f), and 2(g) to Fig. 1(b), one can easily see that

$$
\begin{aligned}
\epsilon_{H_{i}} & =f_{H_{i}}-n_{i} f_{s}, \quad i=1,2, \\
\epsilon_{H_{-i}} & =\left(n_{i}+1\right) f_{s}-f_{L_{i}}, \quad i=1,2, \\
\epsilon_{L_{i}} & =f_{L_{i}}-n_{i} f_{s}, \quad i=1,2, \\
\epsilon_{L_{-i}} & =\left(n_{i}+1\right) f_{s}-f_{H_{i}}, \quad i=1,2 .
\end{aligned}
$$

In deriving (2) and (4), we have used the following relations

$$
\begin{aligned}
\epsilon_{H_{-i}} & =f_{s}-\epsilon_{L_{i}}, \quad i=1,2, \\
\epsilon_{L_{-i}} & =f_{s}-\epsilon_{H_{i}}, \quad i=1,2,
\end{aligned}
$$

which are due to the fact that the relative positions of the replicas ' $i$ ' and ' $-i$ ' in each segment are symmetric with respect to the midpoint of the segment.

Let's consider the neighbor constraint first. From Table I we see that the neighboring relation in Group 1 is $\langle 1,2\rangle$ (or equivalently, $\langle-2,-1\rangle$ ). This relation may be written in a shorthand representation as $\epsilon_{H_{1}} \leq \epsilon_{L_{2}}$ (see Fig. 2(a)), which can lead to $f_{s} \leq \frac{f_{L_{2}}-f_{H_{1}}}{n_{2}-n_{1}}$ with the aid of (1) and (3). Similarly, one can easily find the shorthand representations of the neighbor relations and their corresponding constraints on $f_{s}$ for the other three groups. The result is summarized in Table I. The boundary constraints for each case can be obtained by observing Fig. 2. Taking Case 1 as an example, the neighboring replicas $\langle 1,2\rangle$ must be completely inside the first half of a segment. We therefore see from Fig. 2(a) that the shorthand representations of the boundary constraints for spectra ' 1 ' and ' 2 ' are $\epsilon_{L_{1}} \geq 0$ and $\epsilon_{H_{2}} \leq f_{s} / 2$, respectively, which can result in $f_{s} \leq \frac{f_{L_{1}}}{n_{1}}$ and $f_{s} \geq \frac{f_{H_{2}}}{n_{2}+1 / 2}$ by using (1) and (3). Following the same procedure, it is straightforward to derive the boundary constraints for all other cases. The result 
TABLE II

THE BOUNDARY CONSTRAINTS FOR THE 8 CASES IN FIG. 2.

\begin{tabular}{|c|c|c|c|c|}
\hline Spectrum & Group & Case & Shorthand & Constraint \\
\hline \multirow{4}{*}{ ' 1 ' } & 1 & $\begin{array}{l}1 \\
3\end{array}$ & $\epsilon_{L_{1}} \geq 0$ & $f_{s} \leq \frac{f_{L_{1}}}{n_{1}}$ \\
\hline & 2 & $\begin{array}{l}2 \\
4\end{array}$ & $\epsilon_{H_{-1}} \leq \frac{f_{s}}{2}$ & $f_{s} \leq \frac{f_{L_{1}}}{n_{1}+1 / 2}$ \\
\hline & 3 & $\begin{array}{l}5 \\
7\end{array}$ & $\epsilon_{L_{-1}} \geq 0$ & $f_{s} \geq \frac{f_{H_{1}}}{n_{1}+1}$ \\
\hline & 4 & $\begin{array}{l}6 \\
8\end{array}$ & $\epsilon_{H_{1}} \leq \frac{f_{s}}{2}$ & $f_{s} \geq \frac{f_{H_{1}}}{n_{1}+1 / 2}$ \\
\hline \multirow{4}{*}{ ' 2 ' } & 1 & $\begin{array}{l}4 \\
8\end{array}$ & $\epsilon_{L_{2}} \geq 0$ & $f_{s} \leq \frac{f_{L_{2}}}{n_{2}}$ \\
\hline & 2 & $\begin{array}{l}3 \\
7\end{array}$ & $\epsilon_{H_{-2}} \leq \frac{f_{s}}{2}$ & $f_{s} \leq \frac{f_{L_{2}}}{n_{2}+1 / 2}$ \\
\hline & 3 & $\begin{array}{l}2 \\
6\end{array}$ & $\epsilon_{L_{-2}} \geq 0$ & $f_{s} \geq \frac{f_{H_{2}}}{n_{2}+1}$ \\
\hline & 4 & $\begin{array}{l}1 \\
5\end{array}$ & $\epsilon_{H_{2}} \leq \frac{f_{s}}{2}$ & $f_{s} \geq \frac{f_{H_{2}}}{n_{2}+1 / 2}$ \\
\hline
\end{tabular}

is listed in Table II. Note that the boundary constraints for spectra ' 1 ' and ' 2 ' are listed separately in Table II. For each spectrum $i(i=1,2)$, its boundary constraints for the 8 cases can be categorized into 4 groups. Each group includes 2 cases which share the same boundary constraint.

A valid sampling frequency must satisfy both the neighbor and boundary constraints. By combining Tables I and II, one obtains Table III (here $j=1, k=2$, and $M=2$ ), where the ranges of valid sampling frequency for the 8 cases in Fig. 2 are shown.

\section{BANDPASS SAMPLING $M$-BAND RF SIGNALS}

In this section, the analysis described in Section II is extended to RF signals with an arbitrary number of bands. Consider an $M$-band RF signal shown in Fig. 1(b). When the $M$-band RF signal is sampled at $f_{s} \mathrm{~Hz}$, the spectrum of the sampled signal can be obtained by replicating the spectrum of the original signal at multiples of $f_{s}$. Due to spectral replication, there will be a replica of spectrum ' $i$ ' $(i=1,2, \ldots, M)$ and a replica of spectrum ' $-i$ ' in each segment of the spectrum of the sampled signal. For each pair of replicas ' $i$ ' and ' $-i$ ', there are two choices of ordering them in a segment: placing replica ' $i$ ' in the first half and replica ' $-i$ ' in the second half, or the other way around. Since there are $M$ such pairs of replicas need to be allocated in a segment, the total number of choices is equal to $2^{M}$. To be immune from aliasing, the $M$ replicas allocated to the first half of a segment can not overlap. Hence there are $M$ ! ways of ordering the allocated replicas. Therefore, the total number of possible replica orders in one segment is equal to $2^{M} \times M$ !.

For each possible replica order in the first half of a segment, say, $\left\langle i_{1}, i_{2}, \ldots, i_{M}\right\rangle\left(i_{m} \in\{ \pm 1, \pm 2, \ldots, \pm M\}\right.$ for $m=$ $1,2, \ldots, M)$, there are $M-1$ neighbor constraints on the neighboring relations $\left\langle i_{1}, i_{2}\right\rangle,\left\langle i_{2}, i_{3}\right\rangle, \ldots,\left\langle i_{M-1}, i_{M}\right\rangle$ and 2 boundary constraints on replicas ' $i_{1}$ ' and ' $i_{M}$ '. The neighbor constraint on any neighboring relation, say, $\langle j, k\rangle$, can be written in shorthand as $\epsilon_{H_{j}} \leq \epsilon_{L_{k}}$. The boundary constraint on a replica, say, ' $j$ ', in the first half of a segment is either $\epsilon_{L_{j}} \geq 0$ (if replica ' $j$ ' is ordered the first) or $\epsilon_{H_{j}} \leq \frac{f_{s}}{2}$ (if replica ' $j$ ' is ordered the last). The corresponding constraints on $f_{s}$ for the shorthand representations can then be easily obtained with the aid of (1)-(4). By combining all the neighbor constraints on the neighboring relations $\left\langle i_{1}, i_{2}\right\rangle,\left\langle i_{2}, i_{3}\right\rangle, \ldots$, $\left\langle i_{M-1}, i_{M}\right\rangle$ and the boundary constraints on ' $i_{1}$ ' and ' $i_{M}$ ', one can obtain the range of valid sampling frequency for the replica order $\left\langle i_{1}, i_{2}, \ldots, i_{M}\right\rangle$.

\section{SEARCHING FOR THE RANGES OF VALID SAMPLING FREQUENCY}

The derivation in Sections II and III shows how one can obtain the range of $f_{s}$ for a particular replica order provided that $n_{i}(i=1,2, \ldots, M)$ are given. Since $n_{i}$ is the index of the segment where spectrum ' $i$ ' is located, we have

$$
n_{1}=\left\lfloor\frac{f_{L_{1}}}{f_{s}}\right\rfloor \leq\left\lfloor\frac{f_{L_{1}}}{2\left(B_{1}+B_{2}+\cdots+B_{M}\right)}\right\rfloor
$$

where $\lfloor x\rfloor$ denotes the largest integer less than or equal to $x$, and $B_{1}, B_{2}, \ldots$, and $B_{M}$ are defined in Fig. 1(b). In deriving (7), we have used the fact that the bandwidth of a segment should at least accommodate the $2 M$ nonoverlapping replicas '1', '-1', '2', '-2', ..., ' $M$ ', and ' $-M$ ' (i.e., $f_{s} \geq 2\left(B_{1}+B_{2}+\right.$ $\left.\ldots+B_{M}\right)$ ). In addition, the center frequency of spectrum ' $i$ ' (say, $f_{i}$ ) is bounded by

$$
n_{i} f_{s}<f_{i}<\left(n_{i}+1\right) f_{s} .
$$

Multiplying (8) by $\frac{R_{i}}{f_{s}}$ and using the relation $f_{i+1}=R_{i} f_{i}$ (see Fig. 1(b)), one obtains

$$
R_{i} n_{i}<\frac{f_{i+1}}{f_{s}}<R_{i}\left(n_{i}+1\right), \quad i=1,2, \ldots, M-1
$$

Taking \lfloor\rfloor on (9) and using the fact that $n_{i+1}=\left\lfloor\frac{f_{i+1}}{f_{s}}\right\rfloor$, we have

$$
\left\lfloor R_{i} n_{i}\right\rfloor \leq n_{i+1} \leq\left\lfloor R_{i} n_{i}+R_{i}\right\rfloor, \quad i=1,2, \ldots, M-1 .
$$

We see that the possible values of $n_{i+1}$ can be determined given $n_{i}$.

Based on the above analysis, we may summarize the procedure for obtaining the ranges of valid sampling frequency for $M$-band RF signals as follows. First we choose an appropriate $n_{1}$ using (7), then we choose appropriate $n_{2}, n_{3}, \ldots$, and $n_{M}$ using (10). Given the $\left(n_{1}, n_{2}, \ldots, n_{M}\right)$, we find the neighbor and boundary constraints for each of the $2^{M} \times M$ ! possible replica orders. The combination of all the constraints yields the range of valid sampling frequency. The above procedure (called the standard procedure hereafter) is conceptually straightforward. However, for typical applications, one may find that examining all the possible replica orders eventually ends up with only few valid replica orders which yield nonempty sampling frequency ranges.

An alternative procedure to find the valid replica orders and their corresponding sampling frequency ranges for an $M$-band RF signal is to modify the standard procedure as follows. For a given $\left(n_{1}, n_{2}, \ldots, n_{M}\right)$, we first determine the valid replica orders for every pair of bands in the $M$ bands. Namely, for any pair of bands, say, the $j$-th and $k$-th bands, one can use the procedure described in Section II for dualband RF signals to determine the valid sampling frequency 
TABLE III

THE RANGES OF VALID SAMPLING FREQUENCY FOR THE DUAL-BAND SIGNAL CONSISTING OF THE $j$-TH AND $k$-TH BANDS OF AN $M$-BAND RF SIGNAL.

\begin{tabular}{c|c} 
Case & Range of Valid $f_{s}$ \\
\hline 1 & $\frac{f_{H_{k}}}{n_{k}+\frac{1}{2}} \leq f_{s} \leq \min \left\{\frac{f_{L_{j}}}{n_{j}}, \frac{f_{L_{k}}-f_{H_{j}}}{n_{k}-n_{j}}\right\}$ \\
2 & $\frac{f_{H_{k}}}{n_{k}+1} \leq f_{s} \leq \min \left\{\frac{f_{L_{j}}}{n_{j}+\frac{1}{2}}, \frac{f_{L_{k}}-f_{H_{j}}}{n_{k}-n_{j}}\right\}$ \\
3 & $\frac{f_{H_{j}}+f_{H_{k}}}{n_{j}+n_{k}+1} \leq f_{s} \leq \min \left\{\frac{f_{L_{j}}}{n_{j}}, \frac{f_{L_{k}}}{n_{k}+\frac{1}{2}}\right\}$ \\
4 & $\frac{f_{H_{j}}+f_{H_{k}}}{n_{j}+n_{k}+1} \leq f_{s} \leq \min \left\{\frac{f_{L_{j}}}{n_{j}+\frac{1}{2}}, \frac{f_{L_{k}}}{n_{k}}\right\}$ \\
5 & $\max \left\{\frac{f_{H_{j}}}{n_{j}+1}, \frac{f_{H_{k}}}{n_{k}+\frac{1}{2}}\right\} \leq f_{s} \leq \frac{f_{L_{j}}+f_{L_{k}}}{n_{j}+n_{k}+1}$ \\
6 & $\max \left\{\frac{f_{H_{j}}}{n_{j}+\frac{1}{2}}, \frac{f_{H_{k}}}{n_{k}+1}\right\} \leq f_{s} \leq \frac{f_{L_{j}}+f_{L_{k}}}{n_{j}+n_{k}+1}$ \\
7 & $\max \left\{\frac{f_{H_{j}}}{n_{j}+1}, \frac{f_{H_{k}}-f_{L_{j}}}{n_{k}-n_{j}}\right\} \leq f_{s} \leq \frac{f_{L_{k}}}{n_{k}+\frac{1}{2}}$ \\
8 & $\max \left\{\frac{f_{H_{j}}}{n_{j}+\frac{1}{2}}, \frac{f_{H_{k}}-f_{L_{j}}}{n_{k}-n_{j}}\right\} \leq f_{s} \leq \frac{f_{L_{k}}}{n_{k}}$
\end{tabular}

ranges for all the possible replica orders. The result would be that shown in Table III. Such a table is constructed for each pair of bands in the $M$ bands, hence we have $C_{2}^{M}$ tables like Table III. It is easy to see that an $M$-band replica order, say, $\left\langle i_{1}, i_{2}, \ldots, i_{M}\right\rangle$, is a valid replica order (which yields a nonempty sampling frequency range) only if $\left\langle i_{1}, i_{2}\right\rangle$, $\left\langle i_{2}, i_{3}\right\rangle, \ldots$, and $\left\langle i_{M-1}, i_{M}\right\rangle$ are valid dual-band replica orders. That is, a valid $M$-band replica order must be a concatenation of valid replica orders in those $C_{2}^{M}$ tables like Table III. Therefore, one can consider only those $M$-band replica orders which can be concatenated with the valid dual-band replica orders in the $C_{2}^{M}$ tables instead of all the possible $M$-band replica orders. Suppose the number of the $M$-band replica orders which can be concatenated with the valid dual-band replica orders is small, it is shown in the Appendix that this alternative procedure can be more computationally efficient.

\section{COMPuter Simulation - DiRect Downconversion OF GSM SIGNALS}

To demonstrate the usage of the proposed method, we conducted two simulations on direct downconverting GSM signals [11]. In the first simulation, the SDR receiver front end in Fig. 1(a) with $M=2$ was used to directly downconvert the entire service bands of GSM $900(935-960 \mathrm{MHz})$ and GSM 1800 (1805-1880 MHz) systems. Therefore, the input signal to the ADC is a dual-band RF signal like that shown in Fig. 1(b) with $M=2$, where $f_{1}=947.5, f_{2}=1842.5, B_{1}=25$, and $B_{2}=75 \mathrm{MHz}$.

Given the problem, we utilized the proposed method to find the ranges of valid sampling frequency. The result is shown in Table IV. In obtaining Table IV, we found that there were 12 possible $\left(n_{1}, n_{2}\right)$ 's to be considered. Since Table III demands 35 operations (see Appendix), we see that $12 \times 35=420$ operations were used to obtain Table IV. If the method in [9] were used instead, testing the validity of all the frequencies from 100 to $935 \mathrm{MHz}$ would be required. Even if the tests were conducted in steps of $1 \mathrm{MHz}$, we would still need 835 tests (each needs 12 operations). The number of tests could be reduced to 442 by using the method in [10]. However, the
TABLE IV

THE RANGES OF VALID SAMPLING FREQUENCY FOR THE DUAL-BAND GSM SIGNAL.

\begin{tabular}{c|c|c|c}
$n_{1}$ & $n_{2}$ & Case & Range of Valid $f_{s}(\mathrm{MHz})$ \\
\hline 3 & 7 & 7 & $240.000000 \leq f_{s} \leq 240.666667$ \\
2 & 5 & 7 & $320.000000 \leq f_{s} \leq 328.181818$ \\
2 & 5 & 5 & $341.818182 \leq f_{s} \leq 342.500000$ \\
2 & 5 & 4 & $355.000000 \leq f_{s} \leq 361.000000$ \\
2 & 4 & 6 & $384.000000 \leq f_{s} \leq 391.428571$ \\
2 & 4 & 1 & $417.777778 \leq f_{s} \leq 422.500000$ \\
1 & 3 & 7 & $480.000000 \leq f_{s} \leq 515.714286$ \\
1 & 3 & 5 & $537.142857 \leq f_{s} \leq 548.000000$ \\
1 & 3 & 4 & $568.000000 \leq f_{s} \leq 601.666667$ \\
1 & 2 & 6 & $640.000000 \leq f_{s} \leq 685.000000$ \\
1 & 2 & 3 & $710.000000 \leq f_{s} \leq 722.000000$ \\
1 & 2 & 1 & $752.000000 \leq f_{s} \leq 845.000000$
\end{tabular}

number of involved operations $(442 \times 12=5304)$ would still be way larger than that for the proposed method.

In the second simulation, the SDR receiver front end in Fig. 1(a) with $M=3$ was used to receive signals from the GSM 900, GSM 1800, and GSM 1900 (1930-1990 MHz) systems. Therefore, the input signal to the ADC is a three-band RF signal. Given the received signal, we utilized the procedure described in Section IV to search for ranges of valid sampling frequency. Only 5 ranges of valid sampling frequency were found for all possible $\left(n_{1}, n_{2}, n_{3}\right)$. By taking a closer look, we found that all the 5 ranges have $n_{1}=1$. With such a small value of $n_{1}$, the valid sampling frequencies in the 5 ranges are all higher than about $600 \mathrm{MHz}$. One way to lower the sampling rate is by using BPFs with bandwidths narrower than the GSM service bands in Fig. 1(a) to prefilter the received signal. By varying the center frequencies of the BPFs, any channel in the GSM service bands can be preselected as wanted.

As an example, in this simulation we assumed that three BPFs centering at $f_{1}=936, f_{2}=1806$, and $f_{3}=1931 \mathrm{MHz}$ and having a bandwidth of $2 \mathrm{MHz}$ were used in Fig. 1(a). Hence the resulting signal to the ADC has three bands, where bands 1, 2, and 3 are at 935-937, 1805-1807, and 1930$1932 \mathrm{MHz}$, respectively. Using these parameters and following the procedure described in Section IV, a total of 611 ranges of valid sampling frequency were obtained for all possible $\left(n_{1}, n_{2}, n_{3}\right)$. By examining the 611 valid ranges we found that, although the number of possible replica orders is 48 , the maximum number of replica orders which can be concatenated with the dual-band valid replica orders is $L=14$ for a given $\left(n_{1}, n_{2}, n_{3}\right)$. Using the formulas in the Appendix we see that, in this case, the alternative procedure should be more computationally efficient than the standard procedure. To verify this, both the standard and the alternative procedures were used. The result shows that the alternative procedure indeed requires about $13 \%$ less CPU time than the standard procedure.

\section{CONCLUSION}

In this paper, we have presented an efficient method to determine the ranges of valid bandpass sampling frequency for multiband RF signals. The result can be used to efficiently choose an appropriate bandpass sampling frequency for the ADC in a multiband SDR receiver front end. Note that the 
selection of the sampling frequency could be more flexible if nonuniform sampling is allowed, but this would complicate the design of the direct downconverter. It could be beneficial if the sampling process is synchronized with the carrier of the transmitted RF signal. For example, one might be able to use the digital quadrature sampling technique [12] to simplify the data acquisition process.

There are some practical issues to be considered before using the proposed method. Firstly, in performing bandpass sampling, the analog input bandwidth of the ADC needs to meet the spectral range of the RF signals [3]. This is challenging because the attainable maximum analog input frequency of the ADC is limited by its aperture jitter [4]. Secondly, the relatively large sampling rate used by the proposed scheme suggests that high power dissipation is required [13]. This situation can only be improved through the advances of low power consumption ADC technologies (such as the CMOS technology). Thirdly, since the second-order or third-order product distortions caused by nonlinearities in the system could be downconverted to appear in the desired bands, special care should be taken in choosing the sampling frequency in case that nonlinearities exist. Lastly, the prefiltering RF filters need to have steep rolloffs to provide sufficient attenuation of adjacent channel signals, otherwise aliasing may occur in the sampled signal. The currently available RF filters (surface acoustic wave (SAW) or ceramic) have limited stopband attenuation and are designed for a fixed center frequency and a fixed bandwidth. They are not suitable for the proposed flexible filtering scheme. This might be able to be solved by using a micro-electro-mechanical structure (MEMS) filter in the future [14].

\section{APPENDIX}

It is easy to see that Tables I, II, and III require 7, 12, and 35 operations (adds, divides, or compares) for a given $\left(n_{1}, n_{2}\right)$. For an $M$ bands RF signal, we need to consider $C_{2}^{M}$ neighbor constraint tables like Table I and $C_{2}^{M}$ boundary constraint tables like Table II for a given $\left(n_{1}, n_{2}, \ldots, n_{M}\right)$. These tables require $7 C_{2}^{M}+12 C_{2}^{M}=19 C_{2}^{M}$ operations. In addition, to see whether a particular replica order is valid, we need $M$ compares. Since there are $2^{M} \times M$ ! possible replica orders, we need a total of $M \times 2^{M} \times M$ ! compares for each $\left(n_{1}, n_{2}, \ldots, n_{M}\right)$. The total number of operations required for the standard procedure is thus $M \times 2^{M} \times M !+19 C_{2}^{M}$ for each $\left(n_{1}, n_{2}, \ldots, n_{M}\right)$. If the alternative procedure is used, we need to consider $C_{2}^{M}$ dual-band valid sampling frequency tables like Table III, which requires $35 C_{2}^{M}$ operations. If the number of replica orders which can be obtained by concatenating the valid dual-band replica orders in the $C_{2}^{M}$ tables is $L$ for a given $\left(n_{1}, n_{2}, \ldots, n_{M}\right)$, we will need additional $L \times(2 M-3)$ compares to see whether the $L$ replica orders are valid. The total number of operations required for the alternative procedure is thus $35 C_{2}^{M}+L \times(2 M-3)$ for each $\left(n_{1}, n_{2}, \ldots, n_{M}\right)$. For typical applications, the number $L$ is significantly smaller than the total number of replica orders $2^{M} \times M$ !. Therefore, the alternative procedure is more computationally efficient when $M$ is large. For example, if $M=3$ and $L=14$, the numbers of operations required by the standard and the alternative procedures are 201 and 147, respectively. The difference between the two numbers will in general increase with $M$.

\section{ACKNOWLEDGMENT}

This work was supported in part by the National Science Council under Contract NSC 91-2213-E-019-009.

\section{REFERENCES}

[1] J. Mitola, "The software radio architecture," IEEE Commun. Mag., vol. 33, no. 5, pp. 26-38, May 1995.

[2] R. J. Lackey and D. W. Upmal, "Speakeasy: the military software radio," IEEE Commun. Mag., vol. 33, no. 5, pp. 56-61, May 1995.

[3] J. A. Wepman, "Analog-to-digital converters and their applications in radio receivers," IEEE Commun. Mag., vol. 33, no. 5, pp. 39-45, May 1995.

[4] R. H. Walden, "Performance trends for analog-to-digital converters," IEEE Commun. Mag., vol. 37, no. 2, pp. 96-101, Feb. 1999.

[5] R. G. Vaughan, N. L. Scott, and D. R. White, "The theory of bandpass sampling," IEEE Trans. Signal Processing, vol. 39, no. 9, pp. 1973-1984, Sep 1991.

[6] P. G. Cook and W. Bonser, "Architectural overview of the SPEAKeasy system," IEEE J. Select. Areas Commun., vol. 17, no. 4, pp. 650-661, Apr. 1999.

[7] T. Turletti, H. J. Bentzen, and D. Tennenhouse, "Toward the software realization of a GSM base station," IEEE J. Select. Areas Commun., vol. 17, no. 4, pp. 603-612, Apr. 1999.

[8] K. C. Zangi and R. D. Koilpillai, "Software radio issues in cellular base stations," IEEE J. Select. Areas Commun., vol. 17, no. 4, pp. 561-573, Apr. 1999.

[9] D. M. Akos, M. Stockmaster, J. B. Y. Tsui, and J. Caschera, "Direct bandpass sampling of multiple distinct RF signals," IEEE Trans. Commun., vol. 47, no. 7, pp. 983-988, July 1999.

[10] N. Wong and T.-S. Ng, "An efficient algorithm for downconverting multiple bandpass signals using bandpass sampling," in Proc. IEEE Int. Conf. Commun., Helsinki, Finland, June 2001, vol. 3, pp. 910-914.

[11] Digital Cellular Telecommunications System (Phase 2+); Radio Transmission and Reception (GSM 05.05 Version 8.5.1 Release 1999), ETSI EN 300910 Ver. 8.5.1 (2000-11).

[12] K. C. Ho, Y. T. Chan, and R. Inkol, "A digital quadrature demodulation system," IEEE Trans. Aerospace Electron. Syst., vol. 32, no. 4, pp. 12181227, Oct. 1996.

[13] P. B. Kenington, and L. Astier, "Power consumption of A/D converters for software radio applications," IEEE Trans. Veh. Technol., vol. 49, no. 2, pp. 643-650, Mar. 2000.

[14] W. Tuttlebee, Ed., Software Defined Radio: Enabling Technologies. John Wiley \& Sons Ltd, 2002. 